\title{
Combined gaze palsy of horizontal saccades and pursuit contralateral to a midbrain haemorrhage
}

\author{
J BOLLING, $\dagger$ P J M LAVIN*† \\ From the Departments of Neurology* and Ophthalmology, $†$ Vanderbilt University Medical Center, Nashville, \\ Tennessee, USA
}

SUMMARY A midbrain haemorrhage, revealed by CT, caused a contralateral supranuclear horizontal gaze palsy of both saccades and foveal pursuit. These findings confirm the hypothesis that the supranuclear pathways for pursuit decussate to the contralateral midbrain and then decussate back before innervating the ipsilateral paramedian pontine reticular formation.

The supranuclear pathways for horizontal eye movements are not well defined. Voluntary saccades are generated in the contralateral frontal eye fields, Brodmann's area $8 .^{1-3}$ The major pathways for saccades descend through the corona radiata, anterior limb of the internal capsule and medial cerebral peduncle of the midbrain where they are thought to decussate at the level of the trochlear nucleus before continuing caudally to innervate the horizontal gaze centre in the contralateral paramedian pontine reticular formation. ${ }^{4}$

The supranuclear fibres for pursuit project from the posterior parietal lobe ${ }^{5}$ to the ipsilateral paramedian pontine reticular formation. ${ }^{6}$ Daroff and Hoyt suggested that this projection decussates near the midbrain tegmentum and then again with the frontal projection for saccades, at the pontomesencephalic junction, to innervate the ipsilateral paramedian pontine reticular formation. ${ }^{2}$ The following case report provides clinico-radiographic evidence of this double decussation.

\section{Case report}

A 50 year old white man developed horizontal diplopia in primary gaze. The diplopia disappeared on gaze downward and to the left. Fifteen years earlier he had an unexplained transient right hemiparesis which lasted several weeks.

Address for reprint requests: P J M Lavin, MD, Department of Neurology, 2100 Pierce Avenue, Nashville, Tennessee 37212, USA.

Received 5 August 1986. Accepted 27 September 1986.
Examination revealed uncorrected visual acuity of $6 / 9$ OU. He had anisocoria, the right pupil was $4 \mathrm{~mm}$ and the left $3 \mathrm{~mm}$, but both reacted briskly to light and near accommodation. Ocular motility examination revealed an exotropia in primary position, greater with the left eye fixing. He was unable to look to the right voluntarily or when asked to fixate on a target to his right, or to pursue a rightward moving target beyond the midline. He had a right hypertropia on upgaze. Versions were full to the left and downward. His oculocephalic reflex was intact to the left, but disconjugate to the right with failure of adduction of the left eye (fig 1). Ductions to saccadic and pursuit stimuli revealed limitation of abduction of the right eye (presumed as a result of the right horizontal supranuclear gaze palsy), and impairment of both adduction and elevation of the left eye (fig 1). The left eye also failed to adduct during attempted convergence. Double Maddox rod testing showed no cyclodeviation. Forced ductions showed no restrictions. He had no ptosis; however, levator function was limited to $7 \mathrm{~mm}$ on the left side and $15 \mathrm{~mm}$ on the right. Slit lamp examination, tonometry and dilated fundoscopic examination were normal. Formal visual fields examination (Goldmann perimeter) was normal. General neurological examination revealed a mild right hyperreflexia and a right extensor plantar response, though strength was normal.

Two days later the patient returned to clinic with mild bilateral ptosis and levator function of $6 \mathrm{~mm}$ OU. The pupils were unchanged. A tensilon test was negative. Pharmacological testing for Horner's syndrome was also negative. CT scan demonstrated a focal haemorrhagic infarction in the left midbrain (fig 2).

Clinical Summary The patient presented initially with a partial third nerve palsy and a right horizontal supranuclear gaze palsy for both saccades and foveal pursuit. Although unable to perform voluntary saccades or foveal pursuit to the right he had a rightward disconjugate oculocephalic 

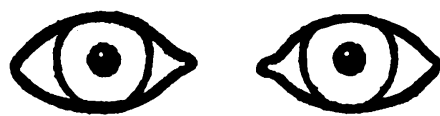

Saccades and pursuit
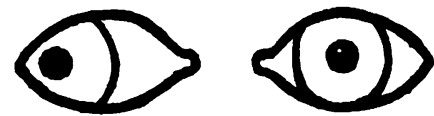

Oculocephalic reflex
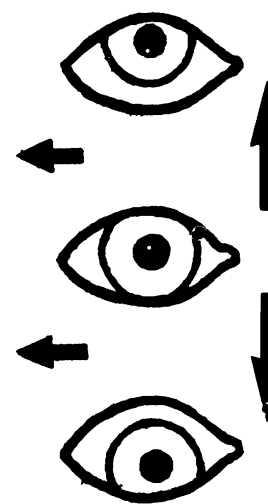

$\uparrow$
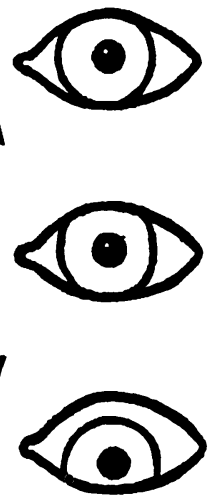

Fig 1 Diagrammatic representation of patient's eye movements.

reflex, demonstrated by abduction of the right eye only (fig 1). He then developed bilateral ptosis. These findings are explained by an ischaemic lesion involving the supranuclear pathways for both horizontal saccades and pursuit as well as part of the left third nerve nuclear complex. The lesion then became haemorrhagic causing more extensive damage to the third nerve nuclear complex.

\section{Discussion}

The patient initially developed an atypical third nerve palsy ${ }^{7}$ and a supranuclear gaze palsy for contralateral horizontal saccades and foveal pursuit, presumably a result of an ischaemic midbrain infarction which later became haemorrhagic, causing more extensive damage to the third nerve nuclear complex. This unusual combination of ocular motor findings is explained by a lesion at the ventral aspect of the third nerve nuclear complex and the midbrain reticular formation ipsilateral to the superior rectus weakness. The lesion, demonstrated by CT (fig 2), was confined to the left side of the midbrain and involved part of the third nerve nuclear complex as well as the supranuclear pathways for both horizontal saccades and foveal pursuit in the midbrain reticular formation remote from the cerebral peduncle.

In 1971 Daroff and Hoyt ${ }^{2}$ suggested that the pursuit pathway decussates in the midbrain to lie with the contralateral saccadic pathway and then decussates again, lower down in the brainstem, with the saccadic pathway in order to innervate the ipsilateral paramedian pontine reticular formation; thus the pursuit pathway must perform a double decussation, at least once. Westheimer and Blair ${ }^{8}$ later demonstrated activation of neurons in the nucleus of the transpeduncular tract (medial terminal nucleus of the accessory optic system located between the substantia nigra and the third nerve fascicle remote from the periaqueductal midbrain reticular formation) during contralateral smooth pursuit in monkeys, supporting the hypothesis that the pursuit pathways cross to the contralateral midbrain. Brigell et al reported an illustrative patient with a thalamic haemorrhage associated with impaired pursuit to the same side and impaired saccades to the side opposite the lesion, ${ }^{9}$ evidence that the pathways for ipsilateral pursuit and contralateral saccades lie close together at the level of thalamus.

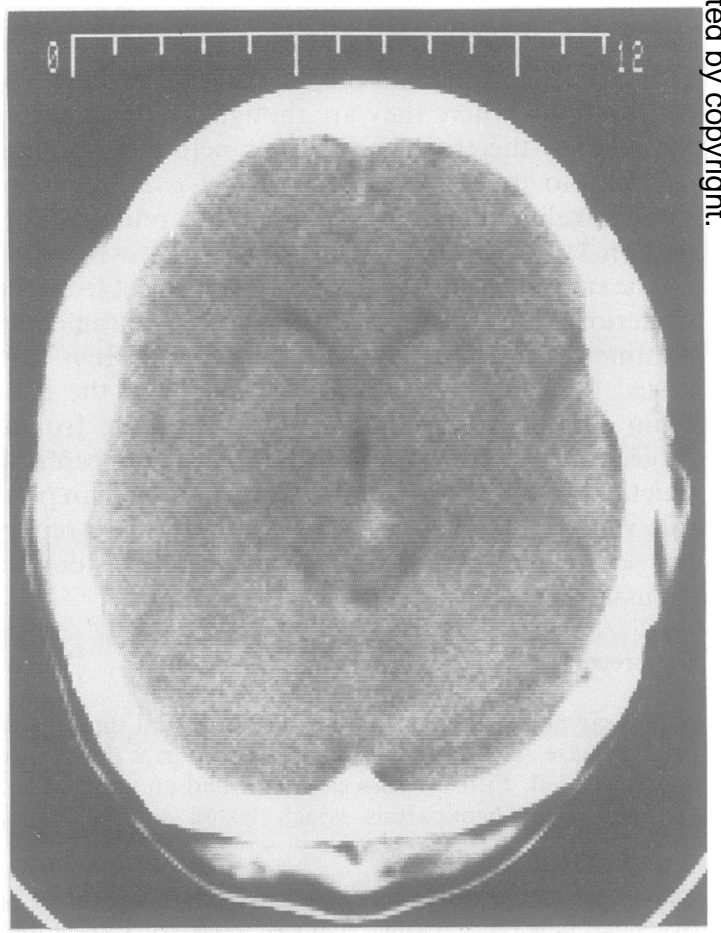

Fig 2 Uncontrasted CT scan demonstrating a left midbrain haemorrhage at the level of the superior colliculus. 
Bril et al described a patient who had "midbrain asterixis" and paralysis of horizontal saccades and pursuit to the same side as a result of a midbrain infarction; ${ }^{10}$ the lesion, however, was described as "discrete bilateral perinuclear" in the rostral midbrain and most likely involved the midbrain reticular formation bilaterally. More recently, Zackon et al reported two patients each with a horizontal gaze palsy, complicating a partial nuclear third nerve palsy, as a result of a midbrain lesion. ${ }^{11}$ The gaze palsies involved both saccades contralateral to, and pursuit ipsilateral to, the third nerve nuclear lesion; the authors concluded that the midbrain reticular formation contains pathways for contralateral saccades and ipsilateral pursuit. However, in their first patient the lesion was bilateral although asymmetric, the gaze palsy was subclinical, detected only by oculographic recordings, and there were lesions in the right frontal and left parietal areas. In their second patient, the gaze palsy was not overcome by oculocephalic manoeuvres, suggesting either diaschisis or a paranuclear pontine lesion. Also, while the lesion was unilateral, it was extensive, in a rostro-caudal fashion, reaching to just above the paramedian pontine reticular formation and probably involving both the ipsilateral fibres for pursuit and the contralateral fibres which may cross in the upper midbrain; indeed their recordings demonstrate bilateral involvement of pursuit.

The report of Pierot-Deseilligny et al suggests that there is a decussation of the smooth pursuit pathways at the pontomesencephalic junction and also in the caudal midbrain based on clinical studies in two patients. ${ }^{12}$ In one patient, however, a pontine haemorrhage caused distortion of the paramedian pontine reticular formations bilaterally, and in the other there was no pathological or radiological correlation to determine the lesion accurately.

In our patient a unilateral midbrain haemorrhage caused impairment of both contralateral saccades and pursuit, documenting the close association of the saccadic and pursuit pathways in the midbrain reticular formation; thus confirming Daroff and Hoyt's hypothesis that there is at least one double decussation of the pursuit pathway. ${ }^{2}$

\section{References}

1 Penfield W, Boldrey E. Somatic motor and sensory representation in the cerebral cortex of man as studied by electrical stimulation. Brain 1937;60:389-443.

2 Daroff RB, Hoyt WF. Supranuclear disorders of ocular control aystems in man: Clinical, anatomical and physiological correlations. In: Bach-y-Rita P, Collins $\mathrm{CC}$, Hyde JE, eds. The Control of Eye Movements. New York: Academic Press, 1971:175-235.

3 Goldberg M, Bushnell MC. Behavioral enhancement of visual responses in monkey cerebral cortex. II. Modulation in frontal eye fields specifically related to saccades. J Neurophysiol 1981;46:773-86.

4 Leichnetz GR. The prefrontal cortico-oculomotor trajectories in the monkey: A possible explanation for the effects of stimulation/lesion experiments on eye movement. J Neurol Sci 1981;49:387-96.

5 Lynch JC, Mountcastle VB, Talbot WH, et al. Parietal lobe mechanisms for directed visual attention. $J$ Neurophysiol 1981;46:773-86.

6 Kestenbaum A. Clinical Methods of Neuroophthalmologic Examination. New York: Grune and Stratton, 1961:195-7, 310, 317.

7 Warren W, Burde RM, Klingele TG, Roper-Hall G. Atypical oculomotor paresis. J Clin Neuro-ophthalmol 1982;2:13-8.

8 Westheimer G, Blair SM. Unit activity in accessory optic system in alert monkeys. Invest Ophthalmol 1974: 533-4.

9 Brigell M, Babikian V, Goodwin JA. Hypometric saccades and low-gain pursuit resulting from a thalamic hemorrhage. Ann Neurol 1984;15:374-8.

10 Bril V, Sharpe JA, Ashby P. Midbrain asterixis. Ann Neurol 1979;6:362-4.

11 Zackon DH, Sharpe JA. Midbrain horizontal gaze paresis. Ann Neurol 1984;16:495-504.

12 Pierrot-Deseilligny C, Chain F, Serdaru M, Gray F, Lhermitte F. The one and a half syndrome. Electrooculographic analysis of five cases with deductions about the physiological mechanisms of lateral gaze. Brain 1981;104:665-9. 